\title{
GENERALIZED IMPLICIT INCLUSION PROBLEMS ON NONCOMPACT SETS WITH APPLICATIONS
}

\author{
SAN-HUA WANG and NAN-JING HUANG ${ }^{凶}$
}

(Received 15 November 2010)

\begin{abstract}
In this paper, a class of generalized implicit inclusion problems is introduced, which can be regarded as a generalization of variational inequality problems, equilibrium problems, optimization problems and inclusion problems. Some existence results of solutions for such problems are obtained on noncompact subsets of Hausdorff topological vector spaces using the famous FKKM theorem. As applications, some existence results for vector equilibrium problems and vector variational inequalities on noncompact sets of Hausdorff topological vector spaces are given.
\end{abstract}

2010 Mathematics subject classification: primary 49J40.

Keywords and phrases: generalized implicit inclusion problem, noncompact set, FKKM theorem, vector equilibrium problem, vector variational inequality.

\section{Introduction}

Let $X$ be a nonempty subset of a Hausdorff topological vector space $E$, and let $f$ : $X \times X \rightarrow R$ be a bifunction such that $f(x, x) \geq 0$ for all $x \in X$. Then the scalar equilibrium problem consists of finding $\bar{x} \in X$ such that

$$
f(\bar{x}, y) \geq 0, \quad \forall y \in X
$$

It provides a unifying framework for many important problems such as optimization problems, variational inequality problems, complementary problems, minimax inequality problems and fixed point problems. It has been widely applied to the study of problems arising in economics, mechanics and engineering (see Blum and Oettli [3]). In recent years, lots of existence results concerning (vector) equilibrium problems and (vector) variational inequalities have been established by many authors in different ways. For more details we refer the reader to [1, 3-5, 8-31] and the references therein.

This work was supported by the National Natural Science Foundation of China $(70831005,10671135)$ and the Natural Science Foundation of Jiangxi Province (2007GZS2120).

(C) 2011 Australian Mathematical Publishing Association Inc. 0004-9727/2011 \$16.00 
In 2000, Di Bella [6] introduced and studied a class of inclusion problems, which we denote by (IP), formulated as follows: find $\bar{x} \in X$ such that

$$
X \subseteq F(\bar{x}),
$$

where $X \subseteq E$ is a nonempty convex subset, and $F: X \rightarrow 2^{E}$ is a multi-valued mapping. By means of the continuous selection theorem and the famous Brouwer fixed point theorem, Di Bella [6] obtained a general existence result for (IP).

THEOREM 1.1 [6, Theorem 1]. Let E be a real Hausdorff topological vector space; $V$ a linear subspace of $E ; X$ a convex subset of $E$, with $\operatorname{ri}(X) \neq \emptyset ; K$ a compact subset of $X ; F: X \rightarrow 2^{E}$ a multi-valued mapping. Further, let $\mathscr{F}$ be a directed (by inclusion) family of finite-dimensional linear subspaces of $V$ meeting $K$, with $V=\bigcup_{S \in \mathscr{F}} S$ and satisfying the following conditions:

(i) for every $S \in \mathscr{F}$ and every compact convex set $Y$, with $K \cap S \subseteq Y \subseteq X \cap S$ and $\operatorname{dim}(Y)=\operatorname{dim}(X \cap S)$, one has $\operatorname{ri}(Y) \backslash F(x) \neq \emptyset$ for all $x \in Y \backslash K$ and $x \notin \operatorname{conv}(\operatorname{ri}(Y) \backslash F(x))$ for all $x \in Y$;

(ii) for every $S \in \mathscr{F}$ and every $y \in(X-X) \cap S$, the set $\{x \in X \cap S: y \in x-F(x)\}$ is closed in $X \cap S$;

(iii) for each $x \in X \cap \bar{V}$ such that $(\operatorname{ri}(X) \cap V) \backslash F(x) \neq \emptyset$, there exist $y_{0} \in \operatorname{ri}(X)$, with $x-y_{0} \in V$ and a neighbourhood $U$ of $x$ such that $z-x+y_{0} \notin F(z)$ for all $z \in U \cap K \cap V$.

Then, there exists $\bar{x} \in K$ such that $\operatorname{ri}(X) \cap V \subseteq F(\bar{x})$.

Di Bella [6] also pointed out that the inclusion problem includes the variational inequality problem as a special case. In fact, the equilibrium problem also can be regarded as a special case of the above inclusion problem by defining $F: X \rightarrow 2^{X}$ by

$$
F(x)=\{y \in X: f(x, y) \geq 0\}, \quad \forall x \in X .
$$

In 2006, Fang and Huang [8] generalized the above inclusion problem to the following extended inclusion problem (EIP), which is formulated to find $\bar{x} \in X$ such that

$$
X \subseteq F(\bar{x}, \bar{x}),
$$

where $X$ is a nonempty closed convex subset of a real Banach space $E$, and $F$ : $X \times X \rightarrow 2^{E}$ is a multi-valued mapping. By means of the auxiliary problem of (EIP) and the well-known Kakutani-Fan-Glicksberg fixed point theorem, Fang and Huang [8] established an existence result for (EIP).

TheOREM 1.2 [8, Theorem 2.3]. Let $X$ be a nonempty, bounded, closed and convex subset of a real reflexive Banach space $E$ and $F: X \times X \rightarrow 2^{E}$ be a multi-valued mapping. Assume that the following conditions hold:

(i) $x \in F(y, x)$ for all $x, y \in X$;

(ii) for each $z \in X$ and each finite-dimensional subspace $D$ of $E$ with $X_{D}=X \cap$ $D \neq \emptyset$, the multi-valued mapping $F^{c}(z, \cdot): X_{D} \rightarrow 2^{E}$ is lower semicontinuous with convex values; 
(iii) for each $z \in X$ and each finite-dimensional subspace $D$ of $E$ with $X_{D} \neq \emptyset$, the set $\left\{x \in X_{D}: X_{D} \subseteq F(z, x)\right\}$ is convex and closed;

(iv) if $\left(x_{\alpha}, z_{\alpha}\right) \in X \times X,\left(x_{\alpha}, z_{\alpha}\right)$ converges to $(x, z) \in X \times X$ weakly, and $X \subseteq$ $F\left(z_{\alpha}, x_{\alpha}\right)$ for all $\alpha$, then $X \subseteq F(z, x)$.

Then there exists $\bar{x} \in X$ such that $X \subseteq F(\bar{x}, \bar{x})$.

Recently, using different methods, several authors further considered inclusion and disclusion problems for multi-valued mappings such as systems of inclusion problems, variational and quasivariational inclusion problems, variational disclusion problems, vector quasivariational inclusion problems and their applications; see, for example, [15, 22, 25, 26, 29] and the references therein.

The main objective of this paper is to present some further findings concerning recent work mentioned above in the area of inclusion problems for multi-valued mappings. We consider the following generalized implicit inclusion problem, denoted (GIIP): find $\bar{x} \in X$ such that

$$
\forall y \in X, \exists v \in T(\bar{x}): y \in F(\bar{x}, v),
$$

where $X$ and $Y$ are two nonempty subsets of two Hausdorff topological vector spaces $E$ and $Z$, respectively, and $T: X \rightarrow 2^{Y}$ and $F: X \times Y \rightarrow 2^{E}$ are two multivalued mappings.

Here are two special cases of (GIIP):

(I) if $E$ and $Z$ are two real Banach spaces, $E=Z, X=Y$, and $T=I$ (identical mapping), then (GIIP) reduces to (EIP) of Fang and Huang [8];

(II) if $E=Z, X=Y, T=I$ and $F(x, y)=F(x)$ for all $x, y \in X$, then (GIIP) reduces to (IP) of Di Bella [6].

In this paper, using the famous Fan-Knaster-Kuratowski-Mazurkiewicz (FKKM) theorem, we prove some existence results for solutions of (GIIP) on noncompact subsets of Hausdorff topological vector spaces. As applications, we show some existence results for vector equilibrium problems and vector variational inequalities on noncompact sets of Hausdorff topological vector spaces.

\section{Preliminaries}

In this section, we recall some definitions and lemmas for later use.

Definition 2.1 [2]. Let $X$ and $Y$ be two topological spaces. A multi-valued mapping $T: X \rightarrow 2^{Y}$ is said to be:

(i) upper semi-continuous (u.s.c.) at $x \in X$ if, for each open set $V$ in $Y$ with $T(x) \subseteq V$, there exists an open neighbourhood $U(x)$ of $x$ such that $T\left(x^{\prime}\right) \subseteq V$ for all $x^{\prime} \in U(x)$;

(ii) lower semi-continuous (1.s.c.) at $x \in X$ if, for each open set $V$ in $Y$ with $T(x) \cap$ $V \neq \emptyset$, there exists an open neighbourhood $U(x)$ of $x$ such that $T\left(x^{\prime}\right) \cap V \neq \emptyset$ for all $x^{\prime} \in U(x)$; 
(iii) u.s.c. (respectively, l.s.c.) on $X$ if it is u.s.c. (respectively, l.s.c.) at every point $x \in X$

(iv) continuous on $X$ if it is both u.s.c. and l.s.c. on $X$;

(v) closed if the graph of $T$ is closed, that is, the set $\operatorname{Gr}(T)=\{(x, y) \in X \times Y: y \in$ $T(x)\}$ is closed in $X \times Y$.

LemMa 2.2 [2]. Let $X$ and $Y$ be two topological spaces, and $F: X \rightarrow 2^{Y}$ a multivalued mapping.

(i) If $F$ is u.s.c. and compact-valued, then $F$ is closed.

(ii) If $F$ is compact-valued, then $F$ is u.s.c. at $x \in X$ if and only if, for any net $\left\{x_{\alpha}\right\} \subseteq X$ such that $x_{\alpha} \rightarrow x$ and for every net $\left\{y_{\alpha}\right\} \subseteq Y$ such that $y_{\alpha} \in F\left(x_{\alpha}\right)$ for all $\alpha$, there exist $y \in F(x)$ and a subnet $\left\{y_{\beta}\right\}$ of $\left\{y_{\alpha}\right\}$ such that $y_{\beta} \rightarrow y$.

DEFINITION 2.3. Let $E$ and $Z$ be two topological vector spaces, $X \subseteq E$ a nonempty convex subset and $C \subseteq Z$ a nonempty convex cone. A multi-valued mapping $F: X \rightarrow$ $2^{Z}$ is said be:

(i) $\quad C$-convex if, for any $x, y \in X$ and $t \in[0,1]$,

$$
F(t x+(1-t) y) \subseteq t F(x)+(1-t) F(y)-C ;
$$

(ii) affine if, for any $x, y \in X(X$ is a vector subspace of $E)$ and $t \in R$,

$$
F(t x+(1-t) y)=t F(x)+(1-t) F(y) .
$$

Definition 2.4 [7]. Let $X \subseteq E$ be a nonempty subset of a vector space $E$. A multi-valued mapping $G: \bar{X} \rightarrow 2^{E}$ is said be a KKM mapping if, for any finite subset $\left\{x_{1}, x_{2}, \ldots, x_{n}\right\} \subseteq X$, one has $\operatorname{co}\left\{x_{1}, x_{2}, \ldots, x_{n}\right\} \subseteq \bigcup_{i=1}^{n} G\left(x_{i}\right)$, where $\operatorname{co}(D)$ denotes the convex hull of $D$.

The following lemma is very important in establishing our main results.

LEMMA 2.5 [7, FKKM theorem]. Let $X$ be a nonempty subset of a Hausdorff topological vector space $E$, and $G: X \rightarrow 2^{E}$ a KKM mapping. If, for any $x \in X, G(x)$ is closed and, for at least one point $x \in X, G(x)$ is compact, then $\bigcap_{x \in X} G(x) \neq \emptyset$.

\section{Main results}

THEOREM 3.1. Let $E$ and $Z$ be two Hausdorff topological vector spaces, $X \subseteq E$ a nonempty closed convex subset and $Y \subseteq Z$ a nonempty subset. Let $T: X \rightarrow 2^{Y}$ and $F: X \times Y \rightarrow 2^{E}$ be two multi-valued mappings. Suppose that:

(i) for any $x \in X$, there exists $v \in T(x)$ such that $x \in F(x, v)$;

(ii) for any $x \in X, v \in T(x)$, the set $\{y \in X: y \notin F(x, v)\}$ is empty or convex;

(iii) for any $y \in X$, the set $\{x \in X: \exists v \in T(x), y \in F(x, v)\}$ is closed in $X$;

(iv) there exists a nonempty compact convex subset $D \subseteq X$ such that, for each $x \in X \backslash D$, there exists some $y_{0} \in D$ such that $y_{0} \notin F(x, v)$ for all $v \in T(x)$. 
Then (GIIP) is solvable, that is, there exists $\bar{x} \in X$ such that

$$
\forall y \in X, \exists v \in T(\bar{x}): y \in F(\bar{x}, v) .
$$

Proof. Define a multi-valued mapping $G: X \rightarrow 2^{D}$ by

$$
G(y)=\{x \in D: \exists v \in T(x), y \in F(x, v)\}, \quad \forall y \in X .
$$

Then it suffices to show that

$$
\bigcap_{y \in X} G(y) \neq \varnothing
$$

Since

$$
G(y)=D \cap\{x \in X: \exists v \in T(x), y \in F(x, v)\},
$$

then, by assumption (iii), it is easy to see that $G(y)$ is closed in $D$. Since $D$ is compact to show (3.1), it suffices to show that the family of sets $\{G(y): y \in X\}$ has the finite intersection property.

For any finite subset $\left\{y_{1}, y_{2}, \ldots, y_{n}\right\} \subseteq X$, let $K=\operatorname{co}\left(D \cup\left\{y_{1}, y_{2}, \ldots, y_{n}\right\}\right)$. Then $K$ is a compact convex subset of $X$. Now we consider the multi-valued mapping $H: K \rightarrow 2^{K}$ defined by

$$
H(y)=\{x \in K: \exists v \in T(x), y \in F(x, v)\}, \quad \forall y \in K .
$$

Since

$$
H(y)=K \cap\{x \in X: \exists v \in T(x), y \in F(x, v)\},
$$

by assumption (iii), it follows that $H(y)$ is closed in $K$, for any $y \in X$.

Next, we shall show that $H$ is a KKM mapping. Suppose that this is not the case. Then there exist $z_{1}, z_{2}, \ldots, z_{m} \in K$ and $t_{i} \geq 0$ for $i=1,2, \ldots, m$ with $\sum_{i=1}^{m} t_{i}=1$ such that $z=\sum_{i=1}^{m} t_{i} z_{i} \notin \bigcup_{i=1}^{m} H\left(z_{i}\right)$. Noting that $K$ is convex, we have $z \in K$. Thus, for every $v \in T(z)$,

$$
z_{i} \notin F(z, v), \quad i=1,2, \ldots, m .
$$

It follows that

$$
z_{i} \in\{y \in X: y \notin F(z, v)\}, \quad i=1,2, \ldots, m .
$$

By assumption (ii), we know that the set $\{y \in X: y \notin F(z, v)\}$ is convex and so $z \in\{y \in X: y \notin F(z, v)\}$, that is, $z \notin F(z, v)$. Since $v \in T(z)$ was arbitrary,

$$
z \notin F(z, v), \quad \forall v \in T(z),
$$

which contradicts assumption (i). Thus $H$ is a KKM mapping.

Since $K$ is compact, it follows from Lemma 2.5 that

$$
\bigcap_{y \in K} H(y) \neq \emptyset \text {. }
$$

Letting $x_{0} \in \bigcap_{y \in K} H(y)$, then

$$
\forall y \in K, \exists v \in T\left(x_{0}\right): y \in F\left(x_{0}, v\right) .
$$


We assert that $x_{0} \in D$. Suppose to the contrary that $x_{0} \notin D$. Then $x_{0} \in K \backslash D \subseteq$ $X \backslash D$. It follows from assumption (iv) that

$$
\exists y_{0} \in D, \forall v \in T\left(x_{0}\right): y_{0} \notin F\left(x_{0}, v\right) .
$$

Since $D \subseteq K$, we can see that (3.4) contradicts (3.3). This shows that $x_{0} \in D$. Notice that $\left\{y_{1}, y_{2}, \ldots, y_{n}\right\} \subseteq K$. It follows from (3.3) that $x_{0} \in \bigcap_{i=1}^{n} G\left(y_{i}\right)$, which implies that the family of sets $\{G(y): y \in X\}$ has the finite intersection property. Hence $\bigcap\{G(y): y \in X\} \neq \emptyset$. This completes the proof.

EXAmple 3.2. Let $E=Z=R$ and $X=Y=R_{+}=[0,+\infty)$. Let $T: X \rightarrow 2^{Y}$ and $F: X \times Y \rightarrow 2^{E}$ be defined, respectively, by $T(x)=[2 x,+\infty)$ and $F(x, v)=[v-$ $x,+\infty)$ for all $x \in X, v \in Y$. Then, we have the following conclusions.

(i) For any $x \in X$, there exists $v=2 x \in T(x)$ such that

$$
x \in[x,+\infty)=[v-x,+\infty)=F(x, v) .
$$

(ii) For any $x \in X, v \in T(x)=[2 x,+\infty)$, the set

$$
\{y \in X: y \notin F(x, v)\}=\left\{y \in R_{+}: y \notin[v-x,+\infty)\right\}=[0, v-x)
$$

is empty when $v=x$ and is convex when $v \neq x$.

(iii) For any $y \in X$, let

$$
H(y)=\{x \in X: \exists v \in T(x), y \in F(x, v)\} .
$$

If $x>y$, then for any $v \in T(x)=[2 x,+\infty)$, we know that $v-x \geq 2 x-x=$ $x>y$. It follows that $y \notin[v-x,+\infty)=F(x, v)$. Thus $x \notin H(y)$; if $x \leq y$, by taking $v=2 x \in T(x)$,

$$
y \in[x,+\infty)=[v-x,+\infty)=F(x, v) .
$$

Thus $x \in H(y)$. Therefore, for each $y \in X, H(y)=[0, y]$, which is closed in $X$.

(iv) Take $D=[0,1]$ and $y_{0}=1$. Then $D \subseteq X$ is compact and $y_{0} \in D$. Moreover, for any $x \in X \backslash D=(1,+\infty), v \in T(x)=[2 x,+\infty)$,

$$
v-x \geq 2 x-x=x>1=y_{0}
$$

and so $y_{0} \notin[v-x,+\infty)=F(x, v)$.

Thus, all the conditions of Theorem 3.1 are satisfied. Now Theorem 3.1 implies that there exists $\bar{x} \in X$ such that

$$
\forall y \in X, \exists v \in T(\bar{x}): y \in F(\bar{x}, v) .
$$

Indeed, we can see that $\bar{x}=0$. 
From Theorem 3.1, we can obtain the following result.

Corollary 3.3. Let $X, Y, E, Z, T, F$ be as given in Theorem 3.1. Suppose that conditions (i), (ii), (iv) of Theorem 3.1 and the following condition are satisfied:

(iii) $^{\prime} T$ and $F$ are u.s.c. with nonempty compact values.

Then (GIIP) is solvable.

PROOF. We need only show that, for each $y \in X$, the set

$$
H(y)=\{x \in X: \exists v \in T(x), y \in F(x, v)\}
$$

is closed in $X$. Indeed, let $\left\{x_{\alpha}\right\} \subseteq H(y)$ be an arbitrary net such that $x_{\alpha} \rightarrow x_{0}$. We need to show that $x_{0} \in H(y)$. Since $\left\{x_{\alpha}\right\} \subseteq X$ and $X$ is closed, $x_{0} \in X$. In addition, for each $\alpha$, there exists $v_{\alpha} \in T\left(x_{\alpha}\right)$ such that

$$
y \in F\left(x_{\alpha}, v_{\alpha}\right)
$$

Since $T$ is u.s.c. with nonempty compact values, it follows from Lemma 2.2(ii) that there exist $v_{0} \in T\left(x_{0}\right)$ and a subnet $\left\{v_{\beta}\right\}$ of $\left\{v_{\alpha}\right\}$ such that $v_{\beta} \rightarrow v_{0}$. Since $F$ is u.s.c. with nonempty compact values, it follows from Lemma 2.2(i) that $F$ is closed. In addition, for any $\beta, y \in F\left(x_{\beta}, v_{\beta}\right)$ and $\left(x_{\beta}, v_{\beta}\right) \rightarrow\left(x_{0}, v_{0}\right)$, so $y \in F\left(x_{0}, v_{0}\right)$. Therefore $x_{0} \in H(y)$, which implies that $H(y)$ is closed. This completes the proof.

From Theorem 3.1, we can obtain the following existence result for a solution to (EIP).

THEOREM 3.4. Let $X$ be a nonempty closed convex subset of a Hausdorff topological vector space $E$, and $F: X \times X \rightarrow 2^{E}$ a multi-valued mapping. Suppose that:

(i) for any $x \in X, x \in F(x, x)$;

(ii) for any $x \in X$, the set $\{y \in X: y \notin F(x, x)\}$ is empty or convex;

(iii) for any $y \in X$, the set $\{x \in X: y \in F(x, x)\}$ is closed in $X$;

(iv) there exists a nonempty compact convex subset $D \subseteq X$ such that, for each $x \in X \backslash D$, there exists some $y_{0} \in D$ such that $y_{0} \notin F(x, x)$.

Then (EIP) is solvable, that is, there exists $\bar{x} \in X$ such that

$$
X \subseteq F(\bar{x}, \bar{x}) .
$$

Proof. Letting $Z=E, Y=X$ and $T=I$ in Theorem 3.1, it is easy to check that all the conditions of Theorem 3.1 are satisfied and so Theorem 3.1 yields the conclusion. This completes the proof.

REMARK 3.5. The extended inclusion problem (EIP) has been studied by Fang and Huang [8]. However, Theorem 3.4 is quite different from Fang and Huang [8, Theorem 2.3] in the following ways:

(a) the space $E$ need not be a Banach space;

(b) the subset $X$ need not be bounded; 
(c) the method of proof is different. In fact, Theorem 3.4 is proved using the famous FKKM theorem, while Fang and Huang [8, Theorem 2.3] is proved using the auxiliary problem of (EIP) and the well-known Kakutani-Fan-Glicksberg fixed point theorem.

ExAmple 3.6. Let $E=R$ and $X=R_{+}=[0,+\infty)$. For any $x, y \in X$, let

$$
F(x, y)= \begin{cases}{[x,+\infty)} & \text { if } x=y, \\ (-1,1) & \text { otherwise }\end{cases}
$$

If we take $D=[0,1]$ and $y_{0}=1$, then $D \subseteq X$ is compact and $y_{0} \in D$. Moreover, by a simple computation, it is easy to see that all the conditions of Theorem 3.4 are satisfied so (EIP) is solvable by Theorem 3.4. Indeed, we can see that $\bar{x}=0$ is a solution. However, we cannot use Fang and Huang [8, Theorem 2.3] to show the solvability of (EIP) since $X$ is unbounded.

From Theorem 3.4, we can obtain the following existence result for a solution of (IP).

THEOREM 3.7. Let X be a nonempty closed convex subset of a Hausdorff topological vector space $E$ and $F: X \rightarrow 2^{E}$ a multi-valued mapping. Suppose that:

(i) for any $x \in X, x \in F(x)$;

(ii) for any $x \in X$, the set $\{y \in X: y \notin F(x)\}$ is empty or convex;

(iii) for any $y \in X$, the set $\{x \in X: y \in F(x)\}$ is closed in $X$;

(iv) there exists a nonempty compact convex subset $D \subseteq X$ such that, for each $x \in X \backslash D$, there exists some $y_{0} \in D$ such that $y_{0} \notin F(x)$.

Then (IP) is solvable, that is, there exists $\bar{x} \in X$ such that

$$
X \subseteq F(\bar{x}) .
$$

Proof. Let $F(x, y)=F(x)$ for all $x, y \in X$ in Theorem 3.4. Then it is easy to check that all the conditions of Theorem 3.4 are satisfied. Thus, Theorem 3.4 yields the conclusion. This completes the proof.

REMARK 3.8. In Theorem 3.7, if $F$ is closed or $F$ is u.s.c. with nonempty compact values, then condition (iii) is satisfied.

REMARK 3.9. The inclusion problem (IP) has been studied by Di Bella [6]. However, Theorem 3.7 is quite different from Di Bella [6, Theorem 1] in the following aspects:

(a) the assumptions are much simpler;

(b) the method of proof is different. In fact, Theorem 3.7 is proved using the famous FKKM theorem, while Di Bella [6, Theorem 1] is proved using the continuous selection theorem and the well-known Brouwer fixed point theorem.

From Theorem 3.7, we can obtain the following result.

THEOREM 3.10. Let $X$ be a nonempty closed convex subset of a Hausdorff topological vector space $E$, and $S: X \rightarrow 2^{X}$ a multi-valued mapping. Suppose that: 
(i) for any $x \in X, S(x)$ is nonempty and convex;

(ii) for any $y \in X$, the set $S^{-1}(y)=\{x \in X: y \in S(x)\}$ is open in $X$;

(iii) there exists a nonempty compact convex subset $D \subseteq X$ such that, for each $x \in X \backslash D$, there exists some $y_{0} \in D$ such that $y_{0} \in S(x)$.

Then $S$ has at least one fixed point in $X$, that is, there exists $\bar{x} \in X$ such that $\bar{x} \in S(\bar{x})$.

PROOF. Suppose that the conclusion is false; then we have

$$
x \notin S(x), \quad \forall x \in X .
$$

Define a multi-valued mapping $F: X \rightarrow 2^{X}$ by

$$
F(x)=X \backslash S(x), \quad \forall x \in X .
$$

It follows that for any $x \in X, x \in F(x)$. Moreover, using the assumptions, it is easy to establish the following:

(a) for any $x \in X$, the set $\{y \in X: y \notin F(x)\}=S(x)$ is nonempty and convex;

(b) for any $y \in X$, the set $\{x \in X: y \in F(x)\}=X \backslash S^{-1}(y)$ is closed in X;

(c) for any $x \in X \backslash D$, there exists some $y_{0} \in D$ such that $y_{0} \notin F(x)$.

Hence, all the conditions of Theorem 3.7 are satisfied and it follows from Theorem 3.7 that there exists $\bar{x} \in X$ such that

$$
X \subseteq F(\bar{x})=X \backslash S(\bar{x}) .
$$

Thus $S(\bar{x})=\emptyset$, which contradicts assumption (i). Therefore, $S$ has at least one fixed point in $X$. This completes the proof.

REMARK 3.11. In Theorem 3.10, if $X$ is a nonempty compact convex subset of $E$, by taking $D=X$, condition (iii) is satisfied automatically and Theorem 3.10 reduces to the classical Fan-Browder fixed point theorem.

The following example shows that Theorem 3.10 is quite different from the classical Fan-Browder fixed point theorem.

EXAmple 3.12. Let $E=R, X=R_{+}=[0,+\infty)$ and $S: X \rightarrow 2^{X}$ be defined by

$$
S(x)=\left[0, x^{3}+\frac{1}{8}\right), \quad \forall x \in X .
$$

If we take $D=[0,1]$ and $y_{0}=1$, then $D \subseteq X$ is compact and $y_{0} \in D$. Moreover, it is easy to check that all the conditions of Theorem 3.10 are satisfied and so Theorem 3.10 shows that $S$ has at least one fixed point in $X$. However, we cannot use the classical Fan-Browder fixed point theorem to show the existence of fixed points for $S$ since $X=[0,+\infty)$ is not compact.

In Theorem 3.1, if $E$ is a real reflexive Banach space, then the compactness of $D$ can be weakened. 
THEOREM 3.13. Let $Y$ and $Z$ be the same as in Theorem 3.1. Let E be a real reflexive Banach space, and $X \subseteq E$ be a nonempty closed convex subset. Let $T: X \rightarrow 2^{Y}$ and $F: X \times Y \rightarrow 2^{E}$ be two multi-valued mappings. Suppose that:

(i) for any $x \in X$, there exists $v \in T(x)$ such that $x \in F(x, v)$;

(ii) for any $x \in X, v \in T(x)$, the set $\{y \in X: y \notin F(x, v)\}$ is empty or convex;

(iii) for any $y \in X$, the set $\{x \in X: \exists v \in T(x), y \in F(x, v)\}$ is weakly closed in $X$;

(iv) there exists a nonempty bounded closed convex subset $D \subseteq X$ such that, for each $x \in X \backslash D$, there exists some $y_{0} \in D$ such that $y_{0} \notin F(x, v)$ for all $v \in T(x)$.

Then (GIIP) is solvable.

Proof. Since $E$ is a real reflexive Banach space, $X \subseteq E$ is a nonempty closed convex subset and $D \subseteq X$ is a nonempty bounded closed convex subset, it follows that $X$ is closed and $D$ is compact with respect to the weak topology of $E$. Thus endowing $E$ with the weak topology, it is easy to see that all the conditions of Theorem 3.1 are satisfied with respect to the weak topology of $E$ and the result follows from Theorem 3.1.

\section{Applications}

In this section we shall apply Theorem 3.1 to present some existence results for vector equilibrium problems and vector variational inequalities on noncompact sets.

\subsection{Weak vector equilibrium problems.}

THEOREM 4.1. Let $E_{1}, E_{2}$ and $Z$ be three Hausdorff topological vector spaces, $X \subseteq E_{1}$ a nonempty closed convex subset and $Y \subseteq E_{2}$ a nonempty subset. Suppose that:

(i) $\quad C: X \rightarrow 2^{Z}$ is a multi-valued mapping such that, for any $x \in X, C(x)$ is a convex cone with nonempty interior, that is, int $C(x) \neq \emptyset$;

(ii) $\quad W: X \rightarrow 2^{Z}$, defined by $W(x)=Z \backslash\{$-int $C(x)\}$ for all $x \in X$, is a closed multi-valued mapping;

(iii) $T: X \rightarrow 2^{Y}$ is u.s.c. with nonempty compact values;

(iv) $g: X \times Y \rightarrow X$ is continuous and $h: X \times X \rightarrow X$ is continuous in the first argument;

(v) $\quad F: X \times X \rightarrow 2^{Z}$ is a multi-valued mapping satisfying the following conditions:

(a) $F$ is u.s.c. with nonempty compact values;

(b) for any $x \in X$, there exists $v \in T(x)$ such that $F(g(x, v), h(x, x)) \nsubseteq$ -int $C(x)$;

(c) for any $x \in X, v \in T(x), F(g(x, v), h(x, y))$ is $C(x)$-convex or affine in $y$;

(d) there exists a nonempty compact convex subset $D \subseteq X$ such that, for each $x \in X \backslash D$, there exists some $y_{0} \in D$ such that $F\left(g(x, v), h\left(x, y_{0}\right)\right) \subseteq$ -int $C(x)$ for all $v \in T(x)$. 
Then, the implicit multi-valued weak vector equilibrium problem is solvable, that is, there exists $\bar{x} \in X$ such that

$$
\forall y \in X, \exists v \in T(\bar{x}): F(g(\bar{x}, v), h(\bar{x}, y)) \nsubseteq-\operatorname{int} C(\bar{x}) .
$$

ProOF. Define a multi-valued mapping $G: X \times Y \rightarrow 2^{X}$ as follows:

$$
G(x, v)=\{y \in X: F(g(x, v), h(x, y)) \not \subset-\operatorname{int} C(x)\}, \quad \forall x \in X, v \in Y .
$$

Then, it is sufficient to show that $T$ and $G$ satisfy all the conditions of Theorem 3.1.

(1) By assumption (v)(b), for any $x \in X$, there exists $v \in T(x)$ such that $x \in G(x, v)$.

(2) For any $x \in X, v \in T(x)$, the set

$$
\{y \in X: y \notin G(x, v)\}=\{y \in X: F(g(x, v), h(x, y)) \subseteq-\operatorname{int} C(x)\}
$$

is empty or convex. Indeed, let $K=\{y \in X: y \notin G(x, v)\}$. Suppose that $K \neq \emptyset$, $y_{1}, y_{2} \in K, t \in(0,1)$, and $y_{t}=t y_{1}+(1-t) y_{2}$. Then $y_{1}, y_{2} \in X$ and

$$
F\left(g(x, v), h\left(x, y_{i}\right)\right) \subseteq-\text { int } C(x), \quad i=1,2 .
$$

Since $X$ is convex, $y_{t} \in X$.

(I) If $F(g(x, v), h(x, y))$ is $C(x)$-convex in $y$, then

$$
\begin{aligned}
& F\left(g(x, v), h\left(x, y_{t}\right)\right) \subseteq t F\left(g(x, v), h\left(x, y_{1}\right)\right) \\
&+(1-t) F\left(g(x, v), h\left(x, y_{2}\right)\right)-C(x) \\
& \subseteq-\operatorname{int} C(x)-\operatorname{int} C(x)-C(x) \\
& \subseteq-\operatorname{int} C(x) .
\end{aligned}
$$

(II) If $F(g(x, v), h(x, y))$ is affine in $y$, then

$$
\begin{aligned}
& F\left(g(x, v), h\left(x, y_{t}\right)\right)= t F\left(g(x, v), h\left(x, y_{1}\right)\right) \\
&+(1-t) F\left(g(x, v), h\left(x, y_{2}\right)\right) \\
& \subseteq-\operatorname{int} C(x)-\operatorname{int} C(x) \\
& \subseteq-\operatorname{int} C(x) .
\end{aligned}
$$

From (4.2), (4.3), we can see that for each $t \in(0,1), y_{t} \in K$. Thus $K$ is convex.

(3) For any $y \in X$, the set

$$
\begin{aligned}
\{x \in X & : \exists v \in T(x), y \in G(x, v)\} \\
& =\{x \in X: \exists v \in T(x), F(g(x, v), h(x, y)) \nsubseteq-\operatorname{int} C(x)\}
\end{aligned}
$$

is closed in $X$. Indeed, let

$$
P=\{x \in X: \exists v \in T(x), y \in G(x, v)\}
$$


and let $\left\{x_{\alpha}\right\} \subseteq P$ be an arbitrary net such that $x_{\alpha} \rightarrow x_{0} \in X$. Then, for each $\alpha$, there exists $v_{\alpha} \in T\left(x_{\alpha}\right)$ such that

$$
F\left(g\left(x_{\alpha}, v_{\alpha}\right), h\left(x_{\alpha}, y\right)\right) \nsubseteq-\operatorname{int} C\left(x_{\alpha}\right) .
$$

Thus, for each $\alpha$, there exists $\omega_{\alpha} \in F\left(g\left(x_{\alpha}, v_{\alpha}\right), h\left(x_{\alpha}, y\right)\right)$ such that

$$
\omega_{\alpha} \notin-\text { int } C\left(x_{\alpha}\right) \text {. }
$$

It follows that

$$
\omega_{\alpha} \in Z \backslash\left\{\text {-int } C\left(x_{\alpha}\right)\right\}=W\left(x_{\alpha}\right), \quad \forall \alpha .
$$

Since $T$ is u.s.c. with nonempty compact values, it follows from Lemma 2.2(ii) that there exist $v_{0} \in T\left(x_{0}\right)$ and a subnet $\left\{v_{\beta}\right\}$ of $\left\{v_{\alpha}\right\}$ such that $v_{\beta} \rightarrow v_{0}$. Further, since $F$ is u.s.c. with nonempty compact values, $g$ is continuous and $h$ is continuous in the first argument, it follows from Lemma 2.2(ii) that there exist $\omega_{0} \in F\left(g\left(x_{0}, v_{0}\right), h\left(x_{0}, y\right)\right)$ and a subnet $\left\{\omega_{\gamma}\right\}$ of $\left\{\omega_{\beta}\right\}$ such that $\omega_{\gamma} \rightarrow \omega_{0}$. Thus $\left(x_{\gamma}, \omega_{\gamma}\right) \rightarrow\left(x_{0}, \omega_{0}\right)$ and, for each $\gamma, \omega_{\gamma} \in W\left(x_{\gamma}\right)$. Since $W$ is closed, it follows that

$$
\omega_{0} \in W\left(x_{0}\right)=Z \backslash\left\{- \text { int } C\left(x_{0}\right)\right\} .
$$

Noting that $\omega_{0} \in F\left(g\left(x_{0}, v_{0}\right), h\left(x_{0}, y\right)\right)$,

$$
F\left(g\left(x_{0}, v_{0}\right), h\left(x_{0}, y\right)\right) \nsubseteq-\operatorname{int} C\left(x_{0}\right) .
$$

Thus $x_{0} \in P$, which implies that $P$ is closed.

(4) By assumption (v)(d), for each $x \in X \backslash D$, there exists some $y_{0} \in D$ such that $y_{0} \notin G(x, v)$ for all $v \in T(x)$.

Thus, all the conditions of Theorem 3.1 are satisfied and it follows from Theorem 3.1 that there exists $\bar{x} \in X$ such that

$$
\forall y \in X, \exists v \in T(\bar{x}): y \in G(\bar{x}, v),
$$

that is,

$$
\forall y \in X, \exists v \in T(\bar{x}): F(g(\bar{x}, v), h(\bar{x}, y)) \nsubseteq \nsubseteq-\operatorname{int} C(\bar{x}) .
$$

This completes the proof.

Corollary 4.2. Let $E_{1}, E_{2}, X, Y, Z$ be the same as in Theorem 4.1. Assume that conditions ( $i$ )-(iv) of Theorem 4.1 and the following conditions are satisfied:

(v) $\quad f: X \times X \rightarrow Z$ is a single-valued mapping satisfying the following conditions:

(a) $f$ is continuous;

(b) for any $x \in X$, there exists $v \in T(x)$ such that $f(g(x, v), h(x, x)) \notin$ -int $C(x)$;

(c) for any $x \in X, v \in T(x), f(g(x, v), h(x, y))$ is $C(x)$-convex or affine in $y$;

(d) there exists a nonempty compact convex subset $D \subseteq X$ such that, for each $x \in X \backslash D$, there exists some $y_{0} \in D$ such that $f\left(g(x, v), h\left(x, y_{0}\right)\right) \in$ -int $C(x)$ for all $v \in T(x)$. 
Then, the implicit weak vector equilibrium problem is solvable, that is, there exists $\bar{x} \in X$ such that

$$
\forall y \in X, \exists v \in T(\bar{x}): f(g(\bar{x}, v), h(\bar{x}, y)) \notin-\operatorname{int} C(\bar{x}) .
$$

REMARK 4.3. The implicit weak vector equilibrium problem has been studied by Chen [4]. Under a suitable monotonicity assumption, Chen [4] obtained some existence results for a solution of the implicit weak vector equilibrium problem using the FKKM theorem. However, in Corollary 4.2, the existence result is established without any monotonicity assumption.

THEOREM 4.4. Let $E$ and $Z$ be two Hausdorff topological vector spaces. Denote by $L(E, Z)$ the space of all continuous linear mappings from $E$ to $Z$ and by $\langle\ell, x\rangle$ the value of $\ell \in L(E, Z)$ at $x \in E$. Let $X \subseteq E$ a nonempty closed convex subset, and $C: X \rightarrow 2^{Z}$ a multi-valued mapping such that, for any $x \in X, C(x)$ is a convex cone with int $C(x) \neq \emptyset$. Take as given the mappings $A: L(E, Z) \rightarrow L(E, Z), f: X \rightarrow$ $Z, g: X \rightarrow X$ and $T: X \rightarrow 2^{L(E, Z)}$. Suppose that:

(i) for any $x \in X$, there exists $v \in T(x)$ such that

$$
\langle A v, x-g(x)\rangle+f(x)-f(g(x)) \notin-\operatorname{int} C(x) ;
$$

(ii) for any $x \in X, f$ is $C(x)$-convex;

(iii) for any $y \in X$, the set

$$
\{x \in X:\langle A v, y-g(x)\rangle+f(y)-f(g(x)) \in-\operatorname{int} C(x), \forall v \in T(x)\}
$$

is open in $X$;

(iv) there exists a nonempty compact convex subset $D \subseteq X$ such that, for each $x \in X \backslash D$, there exists some $y_{0} \in D$ such that

$$
\left\langle A v, y_{0}-g(x)\right\rangle+f\left(y_{0}\right)-f(g(x)) \in-\text { int } C(x) \quad \text { for all } v \in T(x) .
$$

Then the F-implicit weak vector variational inequality is solvable, that is, there exists $\bar{x} \in X$ such that

$$
\forall y \in X, \exists v \in T(\bar{x}):\langle A v, y-g(\bar{x})\rangle+f(y)-f(g(\bar{x})) \notin-\operatorname{int} C(\bar{x}) .
$$

Proof. Let $Y=L(E, Z)$. Define a multi-valued mapping $G: X \times Y \rightarrow 2^{X}$ as follows:

$$
\begin{gathered}
G(x, v)=\{y \in X:\langle A v, y-g(x)\rangle+f(y)-f(g(x)) \notin-\operatorname{int} C(x)\}, \\
\forall x \in X, v \in Y .
\end{gathered}
$$

Then, by an argument similar to Theorem 4.1, we can show that $T$ and $G$ satisfy all the conditions of Theorem 3.1 and it follows from Theorem 3.1 that there exists $\bar{x} \in X$ such that

$$
\forall y \in X, \exists v \in T(\bar{x}): y \in G(\bar{x}, v)
$$


that is,

$$
\forall y \in X, \exists v \in T(\bar{x}):\langle A v, y-g(\bar{x})\rangle+f(y)-f(g(\bar{x})) \notin-\operatorname{int} C(\bar{x}) .
$$

This completes the proof.

REMARK 4.5. Theorem 4.4 is a slight generalization of Lin [24, Theorem 2.2]. For any $x \in X, C(x)$ is assumed to be a proper closed convex pointed cone with apex at the origin in Lin [24, Theorem 2.2], while it is assumed to be a convex cone in the above Theorem 4.4 .

\subsection{Stampacchia vector equilibrium problems.}

THEOREM 4.6. Let $E_{1}, E_{2}$ and $Z$ be three Hausdorff topological vector spaces, $X \subseteq E_{1}$ a nonempty closed convex subset and $Y \subseteq E_{2}$ a nonempty subset. Let $C: X \rightarrow 2^{Z}, T: X \rightarrow 2^{Y}$ and $F: X \times Y \times X \rightarrow 2^{Z}$ be three multi-valued mappings. Suppose that:

(i) for any $x \in X, C(x)$ is a convex cone;

(ii) for any $x \in X$, there exists $v \in T(x)$ such that $F(x, v, x) \nsubseteq-C(x) \backslash\{0\}$;

(iii) for any $x \in X, v \in T(x)$, the set $\{y \in X: F(x, v, y) \subseteq-C(x) \backslash\{0\}\}$ is empty or convex;

(iv) for any $y \in X$, the set $\{x \in X: F(x, v, y) \subseteq-C(x) \backslash\{0\}, \forall v \in T(x)\}$, is open in $X$;

(v) there exists a nonempty compact convex subset $D \subseteq X$ such that, for each $x \in X \backslash D$, there exists some $y_{0} \in D$ such that $F\left(x, v, y_{0}\right) \subseteq-C(x) \backslash\{0\}$ for all $v \in T(x)$.

Then the implicit multi-valued Stampacchia vector equilibrium problem is solvable, that is, there exists $\bar{x} \in X$ such that

$$
\forall y \in X, \exists v \in T(\bar{x}): F(\bar{x}, v, y) \nsubseteq-C(\bar{x}) \backslash\{0\} .
$$

ProOF. Define a multi-valued mapping $G: X \times Y \rightarrow 2^{X}$ as follows:

$$
G(x, v)=\{y \in X: F(x, v, y) \nsubseteq-C(x) \backslash\{0\}\}, \quad \forall x \in X, v \in Y .
$$

Then, by the assumptions, it is easy to see that $T$ and $G$ satisfy all the conditions of Theorem 3.1 and it follows from Theorem 3.1 that there exists $\bar{x} \in X$ such that

$$
\forall y \in X, \exists v \in T(\bar{x}): y \in G(\bar{x}, v)
$$

that is,

$$
\forall y \in X, \exists v \in T(\bar{x}): F(\bar{x}, v, y) \nsubseteq-C(\bar{x}) \backslash\{0\} .
$$

This concludes the proof. 
REMARK 4.7. In Theorem 4.6, if for every $x \in X$ and $v \in T(x), f(x, v, y)$ is $C(x)$ convex or affine in $y$, then condition (iii) is satisfied.

THEOREM 4.8. Let $E$ and $Z$ be two Hausdorff topological vector spaces, and $X \subseteq E$ a nonempty closed convex subset. Let $C: X \rightarrow 2^{Z}$ and $F: X \times X \rightarrow 2^{Z}$ be two multivalued mappings. Suppose that:

(i) for any $x \in X, C(x)$ is a convex cone;

(ii) for any $x \in X, F(x, x) \nsubseteq-C(x) \backslash\{0\}$;

(iii) for any $x \in X, F(x, y)$ is $C(x)$-convex or affine in $y$;

(iv) for any $y \in X$, the set $\{x \in X: F(x, y) \subseteq-C(x) \backslash\{0\}\}$ is open in $X$;

(v) there exists a nonempty compact convex subset $D \subseteq X$ such that, for each $x \in X \backslash D$, there exists some $y_{0} \in D$ such that $F\left(x, y_{0}\right) \subseteq-C(x) \backslash\{0\}$.

Then the multi-valued Stampacchia vector equilibrium problem is solvable, that is, there exists $\bar{x} \in X$ such that

$$
F(\bar{x}, y) \nsubseteq-C(\bar{x}) \backslash\{0\}, \quad \forall y \in X
$$

PROOF. In Theorem 4.6, let $E_{1}=E, E_{2}=Y=\{v\}$ (a singleton set) and, for any $x, y \in X, v \in Y, T(x) \equiv v, F(x, v, y)=F(x, y)$. Then, by the assumptions, it is easy to see that all the conditions of Theorem 4.6 are satisfied and Theorem 4.6 yields the conclusion.

Corollary 4.9. Let $X, E$ and $Z$ be the same as in Theorem 4.8. Let $C \subseteq Z$ be $a$ convex cone and $f: X \times X \rightarrow Z$ a single-valued mapping. Suppose that:

(i) for any $x \in X, f(x, x) \notin-C \backslash\{0\}$;

(ii) for any $x \in X, f(x, y)$ is $C$-convex or affine in $y$;

(iii) for any $y \in X$, the set $\{x \in X: f(x, y) \in-C \backslash\{0\}\}$ is open in $X$;

(iv) there exists a nonempty compact convex subset $D \subseteq X$ such that, for each $x \in X \backslash D$, there exists some $y_{0} \in D$ such that $f\left(x, y_{0}\right) \in-C \backslash\{0\}$.

Then the Stampacchia vector equilibrium problem is solvable, that is, there exists $\bar{x} \in X$ such that

$$
f(\bar{x}, y) \notin-C \backslash\{0\}, \quad \forall y \in X .
$$

REMARK 4.10. Corollary 4.9 generalizes Kazmi and Khan [21, Theorem 2.1] in the following ways:

(a) the spaces $E$ and $Z$ are not necessarily Banach spaces;

(b) the subset $X$ is not necessarily compact;

(c) the cone $C$ is not necessarily solid (that is, int $C \neq \emptyset$ ) or closed or pointed;

(d) for any $x \in X, f(x, x)$ is not necessarily equal to 0 .

Corollary 4.11. Let $X, E, C$ and $Z$ be as given in Corollary 4.9. Let $T: X \rightarrow$ $L(E, Z)$ be a single-valued mapping. Suppose that: 
(i) for any $y \in X$, the set $\{x \in X:\langle T x, y-x\rangle \in-C \backslash\{0\}\}$ is open in $X$;

(ii) there exists a nonempty compact convex subset $D \subseteq X$ such that, for each $x \in X \backslash D$, there exists some $y_{0} \in D$ such that $\left\langle T x, y_{0}-x\right\rangle \in-C \backslash\{0\}$.

Then the Stampacchia vector variational inequality is solvable, that is, there exists $\bar{x} \in X$ such that

$$
\langle T \bar{x}, y-\bar{x}\rangle \notin-C \backslash\{0\}, \quad \forall y \in X .
$$

Proof. Define a mapping $f: X \times X \rightarrow Z$ as follows:

$$
f(x, y)=\langle T x, y-x\rangle, \quad \forall x, y \in X .
$$

Then it is easy to check that $f$ satisfies all the conditions of Corollary 4.9 and it follows from Corollary 4.9 that there exists $\bar{x} \in X$ such that

$$
f(\bar{x}, y) \notin-C \backslash\{0\}, \quad \forall y \in X .
$$

that is,

$$
\langle T \bar{x}, y-\bar{x}\rangle \notin-C \backslash\{0\}, \quad \forall y \in X .
$$

This completes the proof.

REMARK 4.12. Corollary 4.11 generalizes Fang and Huang [9, Theorem 2.1] in the following aspects:

(a) the spaces $E$ and $Z$ are not necessarily Banach spaces;

(b) the subset $X$ is not necessarily compact;

(c) the cone $C$ is not necessarily solid.

\subsection{Strong vector equilibrium problems.}

THEOREM 4.13. Let $E_{1}, E_{2}$ and $Z$ be three Hausdorff topological vector spaces, $X \subseteq E_{1}$ a nonempty closed convex subset and $Y \subseteq E_{2}$ a nonempty subset. Let $C: X \rightarrow 2^{Z}, T: X \rightarrow 2^{Y}$ and $F: X \times Y \times X \rightarrow 2^{Z}$ be three multi-valued mappings. Suppose that:

(i) for any $x \in X, C(x)$ is a convex cone;

(ii) for any $x \in X$, there exists $v \in T(x)$ such that $F(x, v, x) \subseteq C(x)$;

(iii) for any $x \in X, v \in T(x)$, the set $\{y \in X: F(x, v, y) \nsubseteq C(x)\}$ is empty or convex;

(iv) for any $y \in X$, the set $\{x \in X: \exists v \in T(x), F(x, v, y) \subseteq C(x)\}$ is closed in $X$;

(v) there exists a nonempty compact convex subset $D \subseteq X$ such that, for each $x \in$ $X \backslash D$, there exists some $y_{0} \in D$ such that $F\left(x, v, y_{0}\right) \nsubseteq C(x)$ for all $v \in T(x)$.

Then the implicit multi-valued strong vector equilibrium problem is solvable, that is, there exists $\bar{x} \in X$ such that

$$
\forall y \in X, \exists v \in T(\bar{x}): F(\bar{x}, v, y) \subseteq C(\bar{x}) .
$$


ProOF. Define a multi-valued mapping $G: X \times Y \rightarrow 2^{X}$ as follows:

$$
G(x, v)=\{y \in X: F(x, v, y) \subseteq C(x)\}, \quad \forall x \in X, v \in Y .
$$

Then, using the assumptions, it is easy to see that $T$ and $G$ satisfy all the conditions of Theorem 3.1 and it follows from Theorem 3.1 that there exists $\bar{x} \in X$ such that

$$
\forall y \in X, \exists v \in T(\bar{x}): y \in G(\bar{x}, v)
$$

that is,

$$
\forall y \in X, \exists v \in T(\bar{x}): F(\bar{x}, v, y) \subseteq C(\bar{x}) .
$$

This completes the proof.

Similarly, arguing as in the proof of Theorem 4.8, we have the following result.

THEOREM 4.14. Let $E$ and $Z$ be two Hausdorff topological vector spaces, and $X \subseteq E$ a nonempty closed convex subset. Let $C: X \rightarrow 2^{Z}$ and $F: X \times X \rightarrow 2^{Z}$ be two multi-valued mappings. Suppose that:

(i) for any $x \in X, C(x)$ is a convex cone;

(ii) for any $x \in X, F(x, x) \subseteq C(x)$;

(iii) for any $x \in X$, the set $\{y \in X: F(x, y) \nsubseteq C(x)\}$ is empty or convex;

(iv) for any $y \in X$, the set $\{x \in X: F(x, y) \subseteq C(x)\}$ is closed in $X$;

(v) there exists a nonempty compact convex subset $D \subseteq X$ such that, for each $x \in X \backslash D$, there exists some $y_{0} \in D$ such that $F\left(x, y_{0}\right) \nsubseteq C(x)$.

Then the multi-valued strong vector equilibrium problem is solvable, that is, there exists $\bar{x} \in X$ such that

$$
F(\bar{x}, y) \subseteq C(\bar{x}), \quad \forall y \in X .
$$

Corollary 4.15. Let $E$ and $Z$ be two real Banach spaces, $X \subseteq E$ a nonempty closed convex subset, and $C \subseteq Z$ a closed convex cone. Take as given the mappings $T: E \rightarrow L(E, Z)$ and $g: X \rightarrow X$. Suppose that:

(i) for any $x \in X,\langle T x, x-g(x)\rangle \in C$;

(ii) for any $x \in X$, the set $\{y \in X:\langle T x, y-g(x)\rangle \notin C\}$ is empty or convex;

(iii) $T$ and $g$ are continuous on $X$;

(iv) there exists a nonempty compact convex subset $D \subseteq X$ such that, for each $x \in X \backslash D$, there exists some $y_{0} \in D$ such that $\left\langle T x, y_{0}-g(x)\right\rangle \notin C$.

Then the implicit strong vector variational inequality is solvable, that is, there exists $\bar{x} \in X$ such that

$$
\langle T \bar{x}, y-g(\bar{x})\rangle \in C, \quad \forall y \in X .
$$

Proof. Define a mapping $f: X \times X \rightarrow Z$ as follows:

$$
f(x, y)=\langle T x, y-g(x)\rangle, \quad \forall x, y \in X .
$$


Then, by the assumptions, it is easy to check that $f$ satisfies all the conditions of Theorem 4.14 and it follows from Theorem 4.14 that there exists $\bar{x} \in X$ such that

$$
f(\bar{x}, y) \in C, \quad \forall y \in X
$$

that is,

$$
\langle T \bar{x}, y-g(\bar{x})\rangle \in C, \quad \forall y \in X .
$$

This completes the proof.

REMARK 4.16. Corollary 4.15 generalizes Huang and Li [18, Theorem 3.2] in the following aspects:

(a) the subset $X$ is not necessarily a cone;

(b) the mapping $T$ is not necessarily continuous on the whole space $E$.

\section{References}

[1] Q. A. Ansari, W. Oettli and D. Schlager, 'A generalization of vectorial equilibria', Math. Methods Oper. Res. 46 (1997), 147-152.

[2] J. P. Aubin and I. Ekeland, Applied Nonlinear Analysis (Wiley, New York, 1984).

[3] E. Blum and W. Oettli, 'From optimization and variational inequalities to equilibrium problems', Math. Student 63 (1994), 123-145.

[4] X. H. Chen, 'Existences of solution for the implicit multi-valued vector equilibrium problem', J. Appl. Math. Comput. 30 (2009), 469-478.

[5] G. Y. Chen, X. Q. Yang and H. Yu, 'A nonlinear scalarization function and generalized quasivector equilibrium problems', J. Global Optim. 32 (2005), 451-466.

[6] B. Di Bella, 'An existence theorem for a class of inclusions', Appl. Math. Lett. 13 (2000), 15-19.

[7] K. Fan, 'Some properties of convex sets related to fixed point theorems', Math. Ann. 266 (1984), 519-537.

[8] Y. P. Fang and N. J. Huang, 'An existence result for a class of extended inclusion problems with applications to equilibrium problems', J. Anal. Appl. 25 (2006), 257-264.

[9] Y. P. Fang and N. J. Huang, 'Strong vector variational inequalities in Banach spaces', Appl. Math. Lett. 19 (2006), 362-368.

[10] J. Y. Fu, 'Stampacchia generalized vector quasiequilibrium problems and vector saddle points', J. Optim. Theory Appl. 128 (2006), 605-619.

[11] J. Y. Fu and S. H. Wang, 'Stampacchia vector equilibrium problems and vector complementarity problems', Adv. Math. 36(3) (2007), 339-348.

[12] J. Y. Fu, S. H. Wang and Z. D. Huang, 'New type of generalized vector quasiequilibrium problem', J. Optim. Theory Appl. 135 (2007), 643-652.

[13] F. Giannessi, 'Theorems of alternative, quadratic programs and complementarity problems', in: Variational Inequalities and Complementarity Problems (eds. R. W. Cottle, F. Giannessi and J. L. Lions) (Wiley, New York, 1980).

[14] X. H. Gong, 'Symmetric strong vector quasi-equilibrium problems', Math. Methods Oper. Res. 65 (2007), 305-314.

[15] N. X. Hai and P. Q. Khanh, 'Systems of set-valued quasivariational inclusion problems', J. Optim. Theory Appl. 135 (2007), 55-67.

[16] S. H. Hou, H. Yu and G. Y. Chen, 'On system of generalized vector variational inequalities', J. Global Optim. 40 (2008), 739-749.

[17] N. J. Huang and Y. P. Fang, 'On vector variational inequalities in reflexive Banach spaces', J. Global Optim. 32 (2005), 495-505. 
[18] N. J. Huang and J. Li, 'On vector implicit variational inequalities and complementarity problems', J. Global Optim. 34 (2006), 399-408.

[19] N. J. Huang, J. Li and H. B. Thompson, 'Generalized vector $F$-variational inequalities and vector F-complementarity problems for point-to-set mappings', Math. Comput. Modelling 48 (2008), 908-917.

[20] N. J. Huang, J. Li and J. C. Yao, 'Gap functions and existence of solutions for a system of vector equilibrium problems', J. Optim. Theory Appl. 133 (2007), 201-212.

[21] K. R. Kazmi and S. A. Khan, 'Existence of solutions to a generalized system', J. Optim. Theory Appl. 142 (2009), 355-361.

[22] J. K. Kim, Y. P. Fang and N. J. Huang, 'An existence result for a system of inclusion problems with applications', Appl. Math. Lett. 21 (2008), 1209-1214.

[23] I. V. Konnov and J. C. Yao, 'Existence of solutions for generalized vector equilibrium problems', J. Math. Anal. Appl. 233 (2009), 328-335.

[24] Y. C. Lin, 'On F-implicit generalized vector variational inequalities', J. Optim. Theory Appl. 142 (2009), 557-568.

[25] L. J. Lin, C. S. Chuang and S. Y. Wang, 'From quasivariational inclusion problems to Stampacchia vector quasiequilibrium problems, Stampacchia set-valued vector Ekeland's variational principle and Caristi's fixed point theorem', Nonlinear Anal. TMA 71 (2009), 179-185.

[26] L. J. Lin and C. I. Tu, 'The studies of systems of variational inclusions problems and variational disclusions problems with applications', Nonlinear Anal. TMA 69 (2008), 1981-1998.

[27] S. Park, 'Fixed points and quasi-equilibrium problems', Math. Comput. Modelling 34 (2001), 947-954.

[28] B. Ricceri, 'Un théorème d'existence pour les inéquations variationnelles', C. R. Acad. Sci. Paris Sér. I Math. 301 (1985), 885-888.

[29] P. H. Sach and L. A. Tuan, 'Generalizations of vector quasivariational inclusion problems with set-valued maps', J. Global Optim. 43 (2009), 23-45.

[30] S. H. Wang and J. Y. Fu, 'Stampacchia generalized vector quasi-equilibrium problem with setvalued mapping', J. Global Optim. 44 (2009), 99-110.

[31] X. Q. Yang and X. Y. Zheng, 'Approximate solutions and optimality conditions of vector variational inequalities in Banach spaces', J. Global Optim. 40 (2008), 455-462.

SAN-HUA WANG, Department of Mathematics, Sichuan University, Chengdu, Sichuan 610064, PR China

and

Department of Mathematics, Nanchang University, Nanchang, Jiangxi 330031, PR China

NAN-JING HUANG, Department of Mathematics, Sichuan University, Chengdu, Sichuan 610064, PR China

e-mail: nanjinghuang@hotmail.com 